\title{
EL PROCESO DE CONSTRUCCIÓN DE CORPUS TEÓRICOS: LA IMPORTANCIA DE LOS REFERENTES NO TEÓRICOS EN LOS PROCESOS DE TEORIZACIÓN
}

\section{THEORETICAL CORPUS CONSTRUCTION PROCESS: THE IMPORTANCE OF NON THEORETICAL REFERENTS IN THEORETICAL PROCESSES}

Dr. Francisco Covarrubias-Villa (pancheco@prodigy.net.mx) Centro Interdisciplinario de Investigación para el Desarrollo Integral Regional, Unidad Michoacán del Instituto Politécnico Nacional (Michoacán, México).

\begin{abstract}
Every theory is based on a rationality that implies a definition of reality (ontology) and its related epistemological definition; hence every theory perceives certain shapes and contents of reality different from one another. That perceived implies a certain way to construct knowledge, i.e., research objects, test, instruments and hypothesis. The non resolve enigmas in a theory transformed in anomalies and the time comes when scientists create theories with referents of "falsifiable" theories o even discarded ones, articulated with referents coming from non theoretical forms of apprehension of reality such as religion, art and the empirical, creating a new hegemonic theory.
\end{abstract}

Key words: paradigm, theory, anomaly, facts, tests.

\section{Resumen}

Toda teoría está sustentada en una racionalidad que implica una concepción de la realidad (ontología) y su respectiva concepción epistemológica, por lo que cada teoría percibe determinadas formas y contenidos de lo real distintos de los percibidos por otras. Lo percibido implica una manera determinada de construir conocimiento, es decir, objetos de investigación, test, instrumentos e hipótesis. Los enigmas no resueltos en una teoría se convierten en anomalías y llega el momento en el que los científicos formulan teorizaciones con referentes de teorías "falsadas" o simplemente desechadas, articulados con referentes provenientes de modos de apropiación de lo real no teóricos como lo son el religioso, el artístico y el empírico, surgiendo así una nueva teoría hegemónica.

Palabras clave: paradigma, teoría, anomalía, hechos, pruebas. 


\section{Enigmas y anomalías en la teoría}

La construcción de conocimiento científico ha estado signada por procesos de hegemonización de teorías, surgimiento de enigmas y anomalías en la teoría hegemónica, crisis de ésta, surgimiento de otras que plantean soluciones a las anomalías y ascensión de una de ellas al sitio que ocupaba la abandonada. Es Kuhn el primero en plantearlo. Sin embargo, él no aclara plenamente la diferencia entre enigma y anomalía ni el tratamiento y función que cada una tiene en los corpus teóricos; dice: “...a veces, un problema normal, que debería resolverse por medio de reglas y procedimientos conocidos, opone resistencia a los esfuerzos reiterados de los miembros más capaces del grupo dentro de cuya competencia entra. Otras veces, una pieza de equipo, diseñada y construida para fines de investigación normal, no da los resultados esperados, revelando una anomalía que, a pesar de los esfuerzos repetidos, no responde a las esperanzas profesionales. En esas y en otras formas, la ciencia normal se extravía repetidamente. Y cuando lo hace -o sea, cuando la profesión no puede pasar por alto ya las anomalías que subvierten la tradición existente de prácticas científicas - se inician las investigaciones extraordinarias que conducen por fin a la profesión a un nuevo conjunto de compromisos, una base nueva para la práctica de la ciencia" (Kuhn 1986:27).

Para analizar el proceso de paradigmatización de una teoría, es necesario distinguir entre enigmas y anomalías. Cuando una teoría es aceptada por la comunidad científica como solución de una o varias anomalías de la teoría anteriormente hegemónica, aparece como un sistema repleto de enigmas que van siendo resueltos en una práctica investigadora de lo que Kuhn llama "ciencia normal". Pero existen enigmas que no pueden ser resueltos por esa teoría; son anomalías. Éstas no se resuelven porque están percibidas o constituidas con una racionalidad distinta a la del paradigma, debido a que en la conciencia del científico existen referentes de teorías no vigentes, paradigmatizadas en el pasado o no, o por la activación de referentes ateóricos que, evidentemente, no forman parte del corpus teórico en el que es presentada, ni de ningún otro, pero que tienen un fuerte impacto en la percepción de formas y contenidos de lo real que aún no han sido teorizados por los corpus teóricos precedentes.

Popper habla de la falsación de las teorías pero, llevando hasta sus últimas consecuencias lógicas, el planteamiento de Kuhn lo que realmente se hace es construir teorizaciones de anomalías específicas y no de falsar una teoría en su totalidad, si bien la solución de una anomalía podría aparecer como falsación total de la teoría en la que la anomalía se presentó. Frecuentemente la falsación se da dentro de la misma teoría en la que se construyó el enunciado. La pertenencia de dos o más anomalías de una teoría hegemónica a una misma racionalidad puede facilitar la aceleración de su falsación, no así cuando las anomalías pertenecen a racionalidades distintas, pues es necesaria la articulación de dos o más teorizaciones de diferentes anomalías para que se cuestione la validez total de una teoría. Por supuesto que la aceptación científica de la teorización de una anomalía acelera la aplicación de su lógica a la teorización de otras, más ello no garantiza su acierto, dado que su carácter existencial implica una lógica determinada que no puede ser leída por cualquier epistemología, sino sólo por aquella que se desprende de su propia ontología, que no es otra que aquella con la que fue constituida como problema. Efectivamente, se tiende a considerar que las teorías se superan unas a otras. Es decir, que la teoría A es superada por la teoría B y que la A y la B son superadas por la $\mathrm{C}$ y así sucesivamente. La lógica de construcción de conocimiento de la teoría A fue posible por las condiciones materiales históricamente existentes en su momento, que gestaron las condiciones para la generación de la lógica de la teoría $\mathrm{B}$, etcétera. 
Según Kuhn las teorías se paradigmatizan en un proceso en el que paulatinamente se van llenando sus espacios con categorías, conceptos, instrumentos, test, etcétera hasta llegar un momento en el cual todos sus espacios poseen un contenido. Cuando una teoría se ha paradigmatizado surge una nueva que permite pensar otros contenidos, otras formas impensables desde la vieja teoría, iniciándose así un proceso vigoroso que acaba en un nuevo paradigma. Esto no significa que la nueva teoría haya reemplazado a la vieja por haberla superado. La adscripción a una nueva teoría y el abandono de la asumida anteriormente, no necesariamente se da por una superioridad de la nueva. Las teorías se abandonan por la actitud de los científicos de estar a la moda y no necesariamente por el convencimiento de la superioridad de la otra. Es más, podría ser que la paradigmatización de la teoría realmente no suceda en todos los científicos que se adscriben a ella, y que potencialmente sea capaz de considerar nuevos problemas y de enfrentar más desafíos de mayor complejidad que los que ha resuelto. De este modo, la paradigmatización sería un fenómeno que se daría en la conciencia de algunos científicos que asumen un determinado contenido de una teoría y no un problema de la teoría en sí.

Lakatos critica a Kuhn por atribuir a la subjetividad de los científicos la conversión de una teoría a paradigma, argumentando que es necesario recurrir a la racionalidad de las propias teorías y no quedarse en la psique de los científicos. El problema podría enunciarse del siguiente modo: ¿cómo es que los científicos aceptan la teorización de una anomalía como válida? Según Lakatos, para Kuhn es una cuestión de mera subjetividad de los científicos, pero no es así, pues lo cierto es que Kuhn no atiende ese problema porque su preocupación está centrada en demostrar que las teorías no son falsables como supone Popper. Sin embargo, una explicación de ese problema se encuentra efectivamente en la racionalidad con la que las conciencias científicas operan y que acaba siendo la racionalidad de los corpus teóricos.

La aceptación de una propuesta de teorización de una anomalía, no está referida a su grado de correspondencia con los objetos reales aludidos en la anomalía, sino a la presencia de referentes implicantes de su racionalidad en la conciencia de los científicos evaluadores de la teorización. Es decir, que la aceptación del abordaje de un problema de investigación en una "ciencia normal", no depende de la correspondencia existente entre el objeto de investigación construido como problema y los objetos reales aludidos en el objeto de investigación, sino a la presencia en la conciencia de los científicos de los referentes de la teoría a la cual pertenece el problema. El problema se presenta como una relación de correspondencia entre la teorización y lo real, porque tal relación es establecida por los componentes de la conciencia y lo real y no por los componentes de la teorización y lo real pues éste no es otra cosa que lo expresado por la teorización en cuestión. De este modo, a mayor número de referentes correspondientes a su racionalidad, mayores posibilidades de aceptación y de paradigmatización. Sin embargo, desde la antigüedad el problema ha sido planteado como relación entre lo observado y lo pensado, como si la observación fuese independiente del contenido de la conciencia de quien la realiza. "Al reconocer este conflicto entre lo observado y lo pensado, estos primeros filósofos - Heráclito, Parménides, Pitágoras y Empédocles, entre otros - reconocieron que cuando lo natural cambia y se altera, lo hace mediante leyes fijas y universales; en todos ellos está implícita la idea del orden en la naturaleza" (Arrillaga 1987:35-36).

Pero esta forma de pensar no se quedó entre los filósofos de la Grecia clásica sino que es sostenida hasta la actualidad, llevándolas tanto al plano óntico como gnoseológico. "Las leyes -afirma Bunge- son estructuras nómicas (pautas invariantes) al nivel óntico. Las leyes son proposiciones (que a menudo toman la forma de ecuaciones) acerca de pautas objetivas: son pautas al nivel del conocimiento. Las leyes son relaciones invariantes al nivel pragmático: son guías para la acción fundada científicamente. Y las leyes son 
prescripciones metodológicas y/o principios ontológicos (hipótesis acerca de rasgos conspicuos de la realidad)" (Bunge 1969:103).

Obsérvese cómo estas confusiones son compartidas incluso entre supuestos sustentantes de teorías antagónicas; dice Kopnin: "Las hipótesis constituyen el camino indispensable para el descubrimiento de las leyes, para la creación de teorías científicas fidedignas" (Kopnin 1969:10).

Pero Popper plantea lo contrario: “Nuestra propensión a buscar regularidades e imponer leyes a la naturaleza da origen al fenómeno psicológico del pensamiento dogmático o, con mayor generalidad, de la conducta dogmática: esperamos regularidades en todas partes y tratamos de encontrarlas aun allí donde no hay ninguna" (Popper 1972:75). Si las leyes fuesen objetivas, ontológicamente hablando, serían irrefutables y acumulativas, resultando que, al final de cuentas, las leyes serían el único conocimiento acumulado históricamente por la sociedad. Pero no sucede así. Algunas teorías surgen como negación de una ley, si bien esa ley aparece expresada en una teoría y, su falsación como falsación de la teoría y no de la ley.

La mayoría de los filósofos de la ciencia provienen de las ciencias duras y llevan integrado a su conciencia los criterios de cientificidad de la teoría hegemónica de la disciplina científica en la que ellos realizaron sus estudios profesionales. No se trata del método de una disciplina ("el método de la física", "el método de la biología"), ya que tales métodos no existen, sino del método de una teoría adscrito a una disciplina que es elevado a la generalización filosófica, desde la cual se aplican sus criterios a otras teorías de su disciplina y a todas las teorías de todas las disciplinas. No pueden coincidir los criterios de una teoría con los de otra puesto que son distintos y, en ocasiones, contrapuestos o inconmensurables. Lo percibido específicamente en algún supuesto contenido de lo real es llevado a una generalización tal que lo convierte en ley, quedando en el olvido que se trata de un constructo de la razón y no de un contenido real pues, finalmente, lo único con lo que se cuenta es con los contenidos de la conciencia y no con los contenidos de lo real. Esta posición se denomina fenomenológica a diferencia de la que pone acento en los contenidos de lo real, que se denomina realismo.

Para resumir lo expresado en esta primera sección, las teorías nacen, crecen y se desarrollan hasta donde su propia racionalidad lo permite. Poseen un universo cognoscible determinado por la concepción ontológica asumida y ese universo se comporta de conformidad con lo planteado en esa concepción. Asumir una racionalidad determinada implica asumir una perspectiva específica que permite la percepción de determinados aspectos de la realidad y no otros. La teoría que logra convertirse en hegemónica vive un proceso intenso de desarrollo que hace que su corpus rápidamente incorpore conceptos, categorías y conocimiento sustantivo. Entre más completo sea ese corpus teórico, mayor es la posibilidad de percepción de anomalías en su interior. Se entiende por anomalía la existencia de explicaciones insatisfactorias y fenómenos o procesos inexplicables en un corpus teórico. Entre más se acerca un corpus teórico a su integración total, mayor es el número de anomalías percibidas y el malestar de los científicos que asumieron esa teoría como paradigma. Pero ese malestar es vivido de diferentes maneras. Los científicos que más tiempo llevan de haber asumido esa teoría como paradigma se resisten a aceptar la existencia de anomalías, primero y después, se sienten impedidos para formular hipótesis que impliquen soluciones a la anomalía basadas en una concepción teórica distinta. El paradigma asumido por el científico forma parte de su biografía, se ha encarnado en él pues los grandes esfuerzos investigativos, las conferencias, los artículos científicos están relacionados con él, conjuntamente con los ingresos obtenidos por los éxitos logrados. Son 
los científicos más jóvenes los que generan las nuevas teorías por no estar atados a concepciones teóricas determinadas y por no haber participado en la constitución del paradigma en crisis.

\section{Los "hechos" en la teoría}

La conciencia científica se caracteriza por la égida de la lógica del modo teórico de apropiación de lo real, porque en ella predominan numéricamente los referentes de este modo de apropiación o porque algunos referentes integrados a ella son tan potentes que imponen su lógica a la totalidad de la conciencia (Covarrubias 1995:13-153). Es decir, en la conciencia de los científicos predomina la presencia cuantitativa y cualitativa de referentes teóricos, los cuales imponen la lógica con la que opera su conciencia. La conciencia está constituida por referentes de distintos modos de apropiación que son sometidos a la lógica de uno de ellos. Al igual que los corpus teóricos, cada modo de apropiación de lo real posee una lógica determinada intraducible a la de los demás, pero desprendidos del dominio del modo de apropiación de pertenencia e integrados en un universo en el que se articulan con otros provenientes de distintos modos de apropiación al suyo, adquieren la funcionalidad y el sentido que el modo de apropiación dominante les otorga en ese universo. Es así que en la conciencia científica los referentes artísticos, los mágico-religiosos y los empíricos participan activamente en los procesos de construcción de conocimiento científico, bañando a veces con su lógica originaria los constructos cognitivos o transformándose en posibilidad de percepción de formas y contenidos de lo real no consignados por alguna teoría.

Por otra parte, en los procesos de formación académica, los científicos realizan estudios de historia de la ciencia en los que se les informa de la estructura de teorías abandonadas y supuestamente superadas. Con contenidos de esas teorías abandonadas y con percepciones de lo real construidas con referentes no teóricos, los científicos construyen, en ocasiones, problemas irresolubles en la teoría paradigmática en la que ellos mismos realizan su práctica investigadora, los cuales son planteados como enigmas y después como anomalías de esa teoría.

Pero como no necesariamente todas las anomalías provienen de la misma fuente ni están asociadas a la misma racionalidad, es posible que, por lo menos, dos racionalidades sirvan de base al abanico de teorías formuladas para resolverlas, pues con contenidos de conciencia similares a aquellos con los que se formularon las anomalías, se construyen también las teorías que las resuelven.

Es grande la importancia de los referentes no teóricos en los procesos de teorización. Muchos autores reconocen su presencia en la conciencia científica (Paul Feyerabend, Lucien Goldmann, Karel Kosík, Karl R. Popper), si bien no les denominan de esta manera. Los referentes ateóricos son especialmente importantes en los momentos de crisis de la teoría, porque constituyen la condición de posibilidad de construcción de perspectivas novedosas que conduzcan a nuevas teorizaciones de anomalías generadas en la teoría en crisis. En ocasiones influyen solos, en otras (unidos a referentes provenientes de teorías consideradas obsoletas o desechadas en el pasado por metafísicas) los referentes ateóricos operan como perceptor de formas o contenidos de lo real por no pertenecer éstos a teoría alguna.

En realidad, los científicos no trabajan buscando falsar teorías, como lo plantea Popper, sino resolviendo enigmas de la teoría de la que participan o aplicando sus criterios interpretativos para construir conocimiento de otros ámbitos de lo real no teorizados desde ella. Las nuevas teorizaciones no se construyen haciendo "posible elegir entre teorías que ya fueron contrastadas y resultaron falsadas" como pide Feyerabend que sea el método, sino recuperando referentes de esas teorías que, articulados con 
referentes provenientes de modos ateóricos de apropiación y de otros corpus teóricos, hagan posible la constitución de una racionalidad distinta a todas las lógicas y racionalidades de las que los referentes provienen. La propuesta podría consistir en construir nuevas teorías con referentes de teorías falsadas o no y de constructos teóricos o ateóricos ya que, finalmente, en el terreno de la ciencia, nada es falsable pues la interpretación crea el hecho, la inconmensurabilidad de las teorías está a la vista y los instrumentos de mediación entre sujeto y objeto son una prolongación del sujeto hasta el objeto. Por esto es por lo que una teoría nueva puede coincidir en su lógica con una teoría antigua supuestamente superada (Kuhn 1986:142173).

Inmediatamente después de que una nueva teoría es aceptada, se inicia un proceso de abundante y variada construcción de objetos de investigación con la formalidad implicada en la nueva concepción de la realidad y de la construcción de su conocimiento; en la medida en que el tiempo va pasando, se va reduciendo paulatinamente esta actividad, sencillamente porque el número de enigmas existente va desapareciendo, substituyéndose esta actividad por la articulación de conceptos y categorías. Al principio, la teoría aparece como marco general de una interpretación nueva de lo real, vacía de contenido; poco a poco la transformación de los objetos de investigación en conocimiento sustantivo va dándole cuerpo y forma concreta, convirtiendo en objeto de investigación primero "los hechos considerados reveladores de la naturaleza de las cosas", luego, los hechos predichos directamente por la teoría y, por último, las tareas de articulación de los constructos constitutivos del corpus de la teoría (Kuhn 1986:54-57). Esto se debe a que "la descripción de todo hecho particular no sólo es dependiente de alguna teoría que, desde luego, puede ser muy diferente de la teoría que ha de contrastarse, sino que además existen hechos que no pueden descubrirse si no es con la ayuda de alternativas a la teoría que ha de contrastarse, y que dejan de estar disponibles tan pronto como se excluyen tales alternativas" (Feyerabend 2003:22).

Cada teoría surge conteniendo hechos que son diferentes a los contenidos en otras teorías adversarias. Los hechos científicos no son posteriores a una construcción cognitiva pero, aun así, si lo fueran, estarían necesariamente referidos a la teoría que los afirma como existentes. Si una teoría afirma la existencia de un hecho real, los científicos los perciben en la realidad y los investigan con base en lo que la teoría afirma que son y como son. "Cada hecho sólo es comprensible en su contexto, una verdad aún más importante y fundamental, que generalmente se pasa por alto: el concepto mismo del hecho está determinado por la concepción total de la realidad social" (Kosík 1967:65).

Podría suponerse que en el caso de los hechos empíricos percibidos de manera ateórica y convertidos en objeto de las ciencias, es más fácil percibir las diferencias entre teorías que los interpretan. Pero el hecho empírico ateórico tiene un período de vida muy corto pues, en cuanto es considerado objeto de una ciencia es transformado en otra cosa, en un objeto formal de investigación. Dicho con más precisión, los objetos empíricos ateóricos son pretexto para la construcción de objetos formales de investigación. De este modo, el mismo hecho empírico ateórico asumido por teorías contrapuestas, genera, por lo menos, tantos objetos de investigación como teorías lo asumieron, por lo que el ser y la existencia en sí desaparecen y en su lugar quedan las figuras de pensamiento generadas por la ciencia que los aluden pero que son distintos a ellos. El ser y la existencia lo son en cuanto aludidos por la conciencia, adquiriendo la forma y el contenido de la representación construida, más allá de lo que en sí sean. Si esto no fuera así las rupturas epistemológicas serían inexistentes y el conocimiento científico sería acumulativo. A esto se debe la multiplicidad de teorizaciones de los objetos reales dentro de una misma disciplina científica, que incluso hace aparecer al 
mismo objeto real como distinto de sí mismo en sus múltiples representaciones construidas por las diferentes teorías.

Pero a pesar de la complejidad de este problema, para Durkheim "todo objeto de ciencia es una cosa, con excepción, quizá, de los objetos matemáticos" (1996:12). Es decir, los objetos reales son directa e inmediatamente objetos de la ciencia y desaparecen cuando su conocimiento es construido. Pero la realidad no es tan simple. Cada teoría implica determinados objetos de investigación que son ininvestigables desde otra teoría porque ontológicamente se les considera inexistentes.

De este modo, se considera que la ciencia avanza al ir construyendo el conocimiento de más hechos, lo cual permite hablar del "progreso" de la ciencia o de la "acumulación de conocimiento". Por supuesto que sí hay crecimiento del "conocimiento objetivo", como Lakatos lo llama y que mejor sería llamarle conocimiento científico, pero ese crecimiento está limitado a las posibilidades cognitivas de una teoría expresado en el agotamiento de sus enigmas y a la no solución de un conjunto de sus anomalías, más esto no significa que el crecimiento del conocimiento científico se dé por agregación de conocimiento generado en teorías sucesivas. Las teorías están afiliadas a una racionalidad determinada. Cuando en una teoría aparecen enigmas irresolubles, es decir, cuando los enigmas se convierten en anomalías, la teoría entra en crisis y surgen nuevas teorías, convirtiéndose una de ellas en la más abocada a la paradigmatización. Este proceso se reproduce de modo tal que cada racionalidad aparece expresada históricamente en una cadena de teorías, alternándose la paradigmatización en diferentes momentos históricos entre teorías pertenecientes a diferentes racionalidades. De este modo, la solución de enigmas de la teoría en crisis es construida por una teoría que pertenece a la racionalidad en la cual la anomalía fue formulada. Dicho de otra forma, los alcances de una teoría en un momento histórico determinado pueden ser superados por una reconstrucción realizada posteriormente, en la que con nuevos elementos se recupere la racionalidad de aquélla, ampliando notablemente sus horizontes de percepción y solución de anomalías propias no resueltas en el pasado o de anomalías planteadas por otra teoría en crisis.

Bunge cree en la objetividad del conocimiento científico, entendida como relación de correspondencia, por lo menos aproximada, entre lo que se dice de lo real y lo real (Bunge 1969:18); supone que los hechos existen con independencia de la conciencia científica (1969:19, 23, 83-84) y piensa que la racionalidad científica excluye sensaciones, imágenes y pautas de conducta (1969: 17-18). Bunge no alcanza a percibir que las pruebas de verificación son construidas con la misma lógica con la que fue construido el enunciado y menos percibe que, en todo proceso de construcción de conocimiento científico se opera con la lógica de la conciencia del investigador, que no es otra que la lógica de las teorías dominantes en un momento históricosocial, de modo tal que lo percibido y el instrumental usado para la verificación de su objetividad pertenecen a la misma teoría.

Toda teoría incluye en su corpus un conjunto de hechos para cuyo conocimiento se construyen objetos de investigación, se siguen determinados procedimientos para construir el conocimiento de esos supuestos hechos y se usan un conjunto de instrumentos para realizar la investigación. Algunos de esos hechos son consustanciales a una teoría o a un grupo de ellas que poseen una concepción ontológica semejante, otros hechos son percibidos con referentes empíricos, religiosos, artísticos o con referentes procedentes de teorías adversarias. Pero no se trata de que en la realidad exista un menú de hechos perceptibles por todos los sujetos, independientemente de la forma de su conciencia o de la teoría asumida, de modo tal que las diferencias de percepción estuviesen dadas por el modo de su apropiación o por la teoría desde la cual son 
estudiados. Cada teoría contiene un conjunto de hechos: aquellos hechos que son posibles en la ontología asumida por esa teoría pero, como la teoría se encarna en los científicos y los científicos poseen conciencias integradas por referentes de distintos modos de apropiación de lo real y sus referentes teóricos provienen de diversas teorías incluidas las adversarias a la teoría adoptada, algunos de los hechos percibidos en la teoría paradigmatizada acaban finalmente convertidos en anomalías.

La nueva teoría aparece como un enorme arsenal de posibilidades de construcción de conocimiento científico, por la gran cantidad de hechos nuevos que permite percibir. Durante las primeras etapas del proceso de paradigmatización de una teoría existe un amplio universo de objetos por conocer, vínculos que establecer, instrumentos por diseñar y procedimientos que determinar. Es mucho lo que se tiene que construir. Es tan amplio este universo, que sobran ocupaciones para los científicos conversos de la nueva teoría que, ocupados en la ciencia normal, son solucionadores de los enigmas planteados y "no alguien que ponga a prueba los paradigmas. Aunque durante la búsqueda de la solución de un enigma particular puede ensayar una serie de métodos alternativos para abordar el problema descartando los que no le dan los resultados deseados, al hacerlo no está poniendo a prueba al paradigma" (Kuhn 1986:225), sino los enigmas específicos que lo constituyen. En esta labor, los referentes no paradigmáticos son puestos en juego inconsciente e involuntariamente por el investigador y es así cómo, aunque esté realizando su trabajo completamente dentro del paradigma, se llega a formular inconsistencias entre hechos percibidos y principios básicos traducibles a anomalías.

A la crisis de los paradigmas se llega por acumulación de anomalías y agotamiento de enigmas, no por falsación de la teoría. Es desde estos referentes que se diseñan las pruebas para convencer a la comunidad científica de la validez de la teoría asumida. "En las ciencias, la consolidación de la prueba no consiste simplemente, como sucede con la resolución de enigmas, en la comparación de un paradigma único con la naturaleza. En lugar de ello, la prueba tiene lugar como parte de la competencia entre dos paradigmas rivales, para obtener la aceptación por parte de la comunidad científica” (Kuhn 1986:225).

Por supuesto que las pruebas, a pesar de ser presentadas como formulaciones metateóricas en el sentido de afirmar una determinada relación entre lo real y lo teorizado, también forman parte de un corpus y responden a una racionalidad única que es precisamente la asumida por la teoría que se está defendiendo. De este modo, la refutación de un hecho formulado por una teoría, no alcanza al hecho referido por la otra teoría dado que se trata de hechos diferentes, posiblemente reales ónticamente hablando, pero diferencialmente referidos por las teorías. Esto no es percibido por Lakatos cuando afirma que "...las llamadas "refutaciones» no indican un fracaso empírico como Popper ha enseñado, porque todos los programas crecen en un océano permanente de anomalías. Lo que realmente importa son las predicciones dramáticas, inesperadas, grandiosas; unas pocas de éstas son suficientes para decidir el desenlace; si la teoría se retrasa con relación a los hechos, ello significa que estamos en presencia de programas de investigación pobres y regresivos" (Lakatos 1983:15).

Obsérvese cómo Lakatos no se percata de la pertenencia de los hechos a la teoría y por ello otorga tanta importancia a las "predicciones dramáticas", que no son más que hechos construidos con la lógica de una teoría, lo que no le agrega ni un ápice de objetividad, si bien puede con ello incrementar su popularidad entre la comunidad científica. Es aquí donde radica, en buena medida, el carácter relativo de la objetividad del conocimiento científico: los hechos están asociados a la teoría al igual que la manera de estudiarlos. 
La filiación de los hechos a la teoría parece difícil de ser percibido. Como ya se mostró, no la percibe Lakatos, pero tampoco Popper, ni Marx. Popper sostiene: "no podemos prescindir de esta idea de una mejor o peor aproximación a la verdad. Pues no hay duda alguna de que podemos decir, y a menudo decimos, de una teoría $t 2$ que corresponde mejor a los hechos o que, en la medida de nuestro conocimiento, parece corresponder mejor a los hechos, que otra teoría $t 1$ " (Popper 1972:283-284). Cuando se usa como criterio de validez de la teoría su correspondencia con "la medida de nuestro conocimiento", como plantea Popper, lo que en realidad se está haciendo es determinar si la lógica de una teoría corresponde a la lógica de "la medida de nuestro conocimiento", y esa correspondencia no tiene nada que ver con la verdad de lo enunciado por la teoría. Lo que sí se puede hacer es determinar la mayor veracidad de un enunciado con respecto a otro, dentro del mismo corpus teórico, dado que éste es usado como criterio de validación. Colocado en la posición contraria a la de Popper, Marx propone a la práctica como criterio último de objetividad. Dice: "El problema de si al pensamiento humano se le puede atribuir una verdad objetiva, no es un problema teórico, sino un problema práctico. Es en la práctica donde el hombre tiene que demostrar la verdad, es decir, la realidad y el poderío, la terrenalidad de su pensamiento que se aísla de la práctica, es un problema puramente escolástico" (Marx 1971:401).

Por supuesto que la realidad existe con independencia de su reconstrucción teórica y de sus representaciones ateóricas (mágico-religiosa, empírica o artística). El problema está en determinar las condiciones de correspondencia de la representación con lo representado que, como la ciencia y la filosofía, son el único modo de apropiación de lo real que se lo plantea como problema, se ha considerado que el conocimiento construido de manera teórica es el objetivo.

\section{El diseño de pruebas}

Basándose en Kuhn se puede sostener que el diseño de pruebas implica la creación de instrumentos de medición ad hoc. Todo proceso de paradigmatización de una teoría va acompañado del diseño de pruebas y de instrumentos de medición, acordes con la concepción ontológica y epistemológica implicada en la nueva teoría. Inicialmente, los científicos conversos toman los instrumentos que les son útiles de la teoría derrotada y perfeccionan otros de teorías abandonadas que son factibles y de adaptación fácil a los nuevos usos. Más bien son las etapas de crisis cuando mayormente los científicos se dedican a diseñar nuevos instrumentos.

La ciencia actual tiene una gran devoción por la precisión de la medida. Bachelard hace importantes aportaciones en el análisis de este asunto y al respecto dice: "En efecto, una de las exigencias primordiales del espíritu científico, es que la precisión de una medida debe referirse constantemente a la sensibilidad del método de medida y que ha de tener en cuenta naturalmente las condiciones de permanencia del objeto medido. Medir exactamente un objeto fugaz o indeterminado, medir exactamente un objeto fijo y bien determinado con un instrumento grosero, he ahí dos tipos de ocupación vana que la disciplina científica rechaza de inmediato" (Bachelard 2004:250).

Pero aun cuando se mida exactamente un objeto fijo y bien determinado con un fino instrumento, la medida sigue siendo un medio para que la inteligencia construya el conocimiento del objeto. La magnitud no es conocimiento y sin embargo se cree que el conocimiento se torna objetivo en la medida en que se torna instrumental (Bachelard 2004:256). La medición es simplemente un indicador, i.e., una señal, un medio para construir abstracciones del objeto. Sin embargo, la preocupación por la precisión puede convertirse en un obstáculo cognitivo en cuanto impida la abstracción. El instrumento es en realidad teoría "y ha de 
comprenderse que el microscopio es una prolongación del espíritu más que del ojo" (Bachelard 2004:285). Al respecto, Kuhn dice: "En resumen, de manera consciente o no, la decisión de emplear determinado aparato y de usarlo de un modo particular, lleva consigo una suposición de que sólo se presentarán ciertos tipos de circunstancias. Hay expectativas tanto instrumentales como teóricas, y con frecuencia han desempeñado un papel decisivo en el desarrollo científico" (Kuhn 1986:103).

Entre los componentes de un corpus teórico existe una relación que se torna viciada. Las interpretaciones se construyen con base en la concepción onto-epistemológica del corpus teórico asumido; los datos resultaron de la aplicación de instrumentos de investigación construidos con base en la misma concepción; el diseño de pruebas y la interpretación de los resultados, también. Sin embargo, habrá que tomar en cuenta que, una concepción onto-epistemológica determinada, permite percibir aspectos, contenidos y formas de lo real que otros corpus no permiten, aunque esa captación lo sea a la manera que el corpus lo establece, más allá de su correspondencia con lo real. El problema se ubica en que los objetos reales acaban predeterminados por el corpus, es decir, para cada corpus existen determinados objetos reales que son de una determinada manera. Pareciera entonces que estamos ante múltiples realidades coexistentes en dimensiones diferenciales. Cada teoría vencedora es, en el arranque, un marco onto-epistemológico y un programa de construcción de conocimiento que, en la medida que avanza, va fortaleciéndose, resolviendo enigmas, perfeccionando y diseñando instrumentos de medición y pruebas de verificación. Al mismo tiempo, las teorías se van transformando en supuesto existencial y metodológico de los científicos que las sustentan o que se forman en ellas, de modo tal, que acaba convertida en "el modo científico de conocer algo" o en "la ciencia", olvidando que existen otras maneras de hacerlo. Las concepciones de una teoría acaban siendo identificadas con la ciencia por la familiaridad con la que son tratadas por una comunidad epistémica.

Feyerabend percibe parte de este problema al decir que: "Un desarrollo interesante se presenta cuando la ontología defectuosa es comprehensiva, es decir, cuando se piensa que sus elementos están presentes en todos los procesos de cierto dominio. En este caso, toda descripción en el interior del dominio debe ser cambiada y reemplazada por un enunciado diferente (o por ningún enunciado en absoluto)" (2003:271). Se considere que una ontología es válida para un dominio científico específico o para todo el universo, está cumpliendo una función preconcepcional activadora de test, hipótesis e instrumentos que cierran el menú de posibilidades de pensar y conocer lo real entre los científicos. No diferenciar entre objetos de investigación y objetos reales, es no entender adecuadamente el problema de que, un objeto real es aludido o puede ser aludido en múltiples objetos de investigación de la misma disciplina de conocimiento o de diferentes y, no sólo eso, el mismo objeto es apropiado de diferentes modos, no sólo desde distintas disciplinas científicas sino también desde una sola.

Los libros han cumplido un papel preponderante en el proceso de establecimiento de una ontología como supuesto. Los libros son escritos con la racionalidad de la teoría hegemónica del momento y en ellos se presenta el pasado científico como un continuum acumulativo de conocimientos, uno sobre otro hasta llegar al presente en el que se ha accedido por fin a la objetividad. Dice Kuhn al respecto: "Sin embargo, puesto que los libros de texto son vehículos pedagógicos para la perpetuación de la ciencia normal, siempre que cambien el lenguaje, la estructura de problemas o las normas de la ciencia normal, tienen, íntegramente o en parte, que volver a escribirse. En resumen, deben volverse a escribir inmediatamente después de cada revolución científica y, una vez escritos de nuevo, inevitablemente disimulan no sólo el papel desempañado sino también la existencia misma de las revoluciones que los produjeron” (Kuhn 1986: 214). Dice después: "Por razones que son obvias y muy funcionales, los libros de texto científicos (y demasiadas historia antiguas 
de la ciencia) se refieren sólo a las partes del trabajo de científicos del pasado que pueden verse fácilmente como contribuciones al enunciado y a la solución de los problemas paradigmáticos de los libros de texto. [...] En parte por selección y en parte por distorsión, los científicos de épocas anteriores son representados implícitamente como si hubieran trabajado sobre el mismo conjunto de problemas fijos y de acuerdo con el mismo conjunto de cánones fijos que la revolución más reciente en teoría y metodología científicos haya hecho presentar como científicos" (Kuhn 1986:215). Posteriormente señala: "Al disimular esos cambios, la tendencia que tienen los libros de texto a hacer lineal el desarrollo de la ciencia, oculta un proceso que se encuentra en la base de los episodios más importantes del desarrollo científico" (Kuhn 1986:218).

La conciencia teorizante al igual que cualquier otra, está constituida por referentes de distintos modos de apropiación de lo real pero operando bajo la égida del andamiaje constituido por categorías y conceptos. De este modo, existen un conjunto de supuestos existenciales de lo real provenientes de modos ateóricos de apropiación, que participan activamente en la conciencia en los procesos de construcción de conocimiento científico, sin que se sea consciente de ello. Los contenidos ateóricos participan en la conciencia del científico mezclados con los teóricos, integrando una ontología globalmente regida por la racionalidad teórica, pero constitutivamente integrada por una alternancia de dominio de modos de apropiación diferentes en los componentes particulares de la ontología. Esto es percibido por Feyerabend (2003:3) y por Kuhn (1986:212-213). Las concepciones ontológicas implican también supuestos existenciales, metodológicos, teleológicos y axiológicos. Según se suponga que es el mundo, se supone también hacia dónde va y cuál es la función del científico en ese devenir. Todo ello se encarna en los sujetos como supuestos inconscientes, involuntarios y únicos (Kuhn 1986: 284-285). El conjunto integrado por ontología, epistemología, axiología y teleología opera en el científico como sistema orgánico de supuestos que conforman las sensaciones y conducen a la construcción de conocimiento científico bañado de subjetividad inconscientemente asumida como su contrario, como objetividad "científica".

Los objetos generan estímulos y éstos son apropiados de distinto modo por diferentes sujetos a partir de la forma de su conciencia. Los grupos de científicos tienen percepciones similares porque sus conciencias poseen semejante estructura y racionalidad adquirida en los procesos de formación como tales. La formación de científicos no es más que un proceso de incorporación de los referentes compartidos por una comunidad científica. En la "Posdata", Kuhn acaba deslindándose de la inconmensurabilidad planteando la traducibilidad y el entendimiento por los partidarios de otra teoría. El problema sigue en pie: ¿son traducibles las lógicas? No, pero sí son inteligibles. Si fuesen ininteligibles no habría procesos de aceptación de otras teorías y el abandono de paradigmas.

La aceptación de una teoría conlleva una determinada concepción de los objetos reales aludidos en el objeto de estudio de la disciplina científica, inscritos en una determinada concepción ontológica general que, como ya se planteó, pareciera tratarse de realidades diferentes cuando se pasa de un corpus teórico a otro. Planteado a la inversa, puede sostenerse también que los lenguajes son maneras diferenciales de concebir el mundo. Las concepciones se expresan en lenguajes y muchos de los principios básicos de la teoría son aceptados sin que exista la necesidad de expresarlos lingüísticamente, apareciendo en la conciencia de los científicos como presupuestos cognitivos que son activados a la hora de construir conocimiento.

Aprender un lenguaje es aprender una concepción del mundo y de la construcción de su conocimiento que, en el caso de los científicos no siempre aparece explícitamente como ontología sino como supuestos ónticos, a veces, inconscientes. Aunque puedan existir múltiples concepciones onto-epistemológicas entre 
las diferentes disciplinas científicas y dentro de cada una de ellas, lo cierto es que una es predominante y las demás subalternas. La concepción ontológica dominante en una disciplina científica, casi siempre es acorde con la dominante en las demás, esto, gracias al proceso mediante el cual, la racionalidad de una teoría invade los territorios cognitivos de disciplinas científicas diferentes a aquella en la que se generó, convirtiéndose en una metarracionalidad ontológica.

Por lo que a metodología se refiere, cada teoría en proceso de paradigmatización va construyendo también su epistemología en forma de procedimientos, test, instrumentos, sistemas, etcétera que van siendo asumidos como supuestos y tomados poco a poco como condición única de objetividad y de racionalidad científica.

Es casi imposible que un no iniciado paradigmático permanezca por un largo período en una comunidad científica. En México, por ejemplo, existe un organismo público encargado de promover, estimular y velar por el crecimiento y purificación de la comunidad científica. Sólo se apoya la formación de investigadores cuando los más destacados científicos de ese campo determinan que el programa de posgrado está capacitado para formar sujetos de conformidad con el paradigma del momento. Sólo son nombrados investigadores nacionales aquellos sujetos que, a juicio de los científicos más destacados en un campo, reúne las características que el paradigma establece. La inmensa mayoría de las revistas científicas, sólo publican artículos que reúnen los requisitos establecidos por el paradigma, a juicio de los científicos más destacados de ese campo. Cuando un científico escribe un artículo en el que se aborda un problema no considerado por el paradigma, es rechazado por los comités editoriales por tratarse de un asunto acientífico de carácter metafísico, de una lucubración o, de plano, de una locura (Kuhn 1986:259-260).

La organización de comunidades científicas con base en una teoría paradigmática, conlleva la construcción de un lenguaje con la misma velocidad con la que la teoría se convierte en paradigma. Instrumentos, test, reactivos, hipótesis, supuestos, leyes, etcétera, llegan a integrar un lenguaje inteligible sólo para los partícipes del paradigma, llegándose al punto de que ni siquiera es inteligible por los científicos del mismo dominio pero que participan de otro paradigma. El lenguaje es el medio para la radicación de concepciones y prácticas investigativas determinadas o, dicho de otro modo, del proceso de interiorización y encarnación individual de una teoría paradigmatizada. El científico ideal de una teoría paradigmática es aquel que está convencido de que la realidad es como lo dice esa teoría; que la manera de construir conocimiento científico es la utilizada por esa teoría; que la máxima objetividad lograble es la lograda por esa teoría. Al científico paradigmático le interesa comunicarse exclusivamente con sus colegas partícipes del mismo paradigma teórico que él; el resto de la sociedad no le interesa ya que su vida se desarrolla principalmente dentro de los límites del círculo integrado por la comunidad científica a la que pertenece.

La integración al lenguaje es un proceso de incorporación necesaria a un paradigma. El científico investiga los enigmas de una teoría, utiliza las herramientas teóricas y prácticas de esa teoría, construye hipótesis con base en el conocimiento que de su objeto de investigación posee la teoría, etcétera y, evidentemente, usa los vocablos de esa teoría para designar todo lo señalado. El científico lee revistas especializadas en las que aparecen la solución de enigmas de una teoría, los nuevos problemas que desde esa teoría se perciben y el uso de nuevo instrumental; asiste a reuniones científicas en las que presenta ponencias a sus colegas de línea y paradigma de investigación y a conferencias de especialistas en su línea y paradigma de investigación en las que oye los problemas que enfrentan y las soluciones encontradas. Si no está familiarizado con el lenguaje altamente especializado, no podrá comunicarse con sus colegas. 
Por otra parte, en la comunicación con sus colegas no necesita explicar los fundamentos ontoepistemológicos de su planteamiento, sino que va directamente a la exposición de lo descubierto, lo observado o lo cuestionado. Se presentan normalmente "artículos breves dirigidos sólo a los colegas profesionales, a los hombres cuyo conocimiento del paradigma compartido puede presumirse y que son los únicos capaces de leer los escritos a ellos dirigidos" (Kuhn 1986:47). El científico no sólo se aparta del vulgo sino también de las comunidades científicas de otros dominios del conocimiento y de los partícipes de otros paradigmas dentro de su propio dominio. El cerco se va estrechando: no bastó la fragmentación cognitiva especializadora en el proceso de formación de los científicos, ni su carácter paradigmatizador, sino que al científico se le aleja de sus colegas con los que comparte la misma área de dominio, por el simple hecho de que participan de otro paradigma teórico.

Esta situación conduce a preguntarse si existe salida. Dice Feyerabend: “El científico que esté interesado en el máximo contenido empírico, y que desee comprender todos los aspectos posibles de su teoría, tendrá que adoptar, en consecuencia, una metodología pluralista, tendrá que comparar teorías con teorías, en lugar de hacerlo con la 'experiencia', 'datos', o 'hechos'; y tendrá que esforzarse por mejorar, en lugar de eliminarlos, los puntos de vista que parezcan perder en la competición" (Feyerabend 2003:31).

Por esto es por lo que se recomienda que, en el proceso de investigación de un problema, se recopile toda la información posible del conocimiento científico construido de un objeto, más allá de las inclinaciones teóricas del investigador. Pero no basta con recopilar el "conocimiento científico" construido, porque cada paradigma se autoconcibe como el único científico y todas las demás teorías dejan de serlo hayan sido paradigmatizadas o no, tomadas en cuenta o desechadas. También habrá que considerar el conocimiento mágico-religioso o empírico construido desde la lógica de su construcción y de su estructura y no como simple saber.

\section{Bibliografía}

Arrillaga, T. 1987. La naturaleza del conocer. Buenos Aires: Paidós.

Bachelard, G. 2004. La formación del espíritu científico. México: Siglo XXI.

Bunge, M. A. 1969. La ciencia. Buenos Aires: Siglo Veinte.

Covarrubias Villa, F. 1995. La teorización de procesos histórico-sociales. México: UPN.

Durkheim, E. 1996. Las reglas del método sociológico. México: Coyoacán.

Feyerabend, P. 2003. Tratado contra el método. Madrid: Tecnos.

Kosík, K. 1967. Dialéctica de lo concreto. México, Grijalbo.

Kopnin, P. V. 1969. Hipótesis y verdad. México: Grijalbo.

Kuhn, T. S. 1986. La estructura de las revoluciones científicas. México: FCE.

Lakatos, I. 1983. La metodología de los programas de investigación científica. Madrid: Alianza Editorial.

Marx, K. 1971. Tesis sobre Feuerbach. En: Obras Escogidas Moscú: Progreso, Tomo II, pp. 401-403. 
Popper, K. R. 1972. Conjeturas y refutaciones. Barcelona: Paidós.

Recibido el 25 Ago 2009

Aceptado el 18 Ene 2010 